\title{
Recuperación natural del bosque siempreverde afectado por tala rasa y quema en la Reserva Costera Valdiviana, Chile
}

\author{
Natural recovery of the evergreen forest affected by clearcutting and burning \\ in the Valdivian Coastal Reserve, Chile
}

\author{
Mario Romero-Mieres ${ }^{\text {ab*, }}{ }^{\text {Mauro E González }}{ }^{\text {a }}$, Antonio Lara ${ }^{\text {a }}$ \\ *Autor de Correspondencia: Universidad Austral de Chile, Facultad de Ciencias Forestales y Recursos Naturales, \\ Escuela de Graduados, tel.: 56- 63-2221491 maromero@uct.cl \\ ${ }^{a}$ Universidad Austral de Chile, Facultad de Ciencias Forestales y Recursos Naturales, \\ Instituto de Conservación, Biodiversidad y Territorio, Laboratorio Ecología de Bosques, Valdivia, Chile. \\ b Universidad Católica de Temuco, Facultad de Recursos Naturales, Escuela de Ciencias Ambientales, Temuco, Chile.
}

\section{SUMMARY}

This study aimed at understanding the natural recovery of the evergreen forest a decade after being affected by clearcutting and burning, in a site located in the Valdivian Coastal Reserve (3956' S-7340' W). A total of 27 circular (50.2 m²) plots with eight subplots each were established. Floristic composition was recorded and quantitative, diametric and age structures were determined. The results show a richness of 77 vascular species (84.4\% natives), with Lophosoria quadripinnata presenting the highest relative cover (20.7 \%). The presence of hemicryptophytes (35\%) indicates human intervention in the place. Trees were the main form of growth, being Drimys winteri, Saxegothaea conspicua and Amomyrtus luma the species with higher plant density. The latter two species growing under Chusquea macrostachya and Lophosoria quadripinnata cover. Drimys winteri and Embothrium coccineum were established immediately after the anthropogenic disturbance of clearcutting and burning, confirming the pioneering character of these two species after highly severe disturbances. Regeneration of Nothofagus nitida was scarce in the area, probably associated with low availability and seed dispersal capacity from surrounding forests. Understanding the early response of evergreen forests affected by anthropogenic disturbance is very important for assisting and guiding the ecological restoration of these forest ecosystems.

Key words: evergreen forest, clearcutting, burning, floristic diversity.

\section{RESUMEN}

El presente estudio tuvo como objetivo comprender la recuperación natural del bosque siempreverde afectado por tala rasa, quema y abandono hace más de una década en un sitio ubicado en la Reserva Costera Valdiviana (39 56’ S - 73 40’ O). Se establecieron 27 parcelas circulares $\left(50,2 \mathrm{~m}^{2}\right)$ y ocho subparcelas en cada una de ellas, registrando la composición florística y determinando la estructura cuantitativa, diamétrica y etárea. Los resultados indican una riqueza de 77 especies de plantas vasculares (84,4 \% nativas), donde Lophosoria quadripinnata presenta la mayor cobertura relativa (20,7 \%). La presencia de un $35 \%$ de hemicriptófitas evidencia el fuerte impacto antrópico ocurrido en el área de estudio. La principal forma de crecimiento es la arbórea, donde Drimys winteri, Saxegothaea conspicua y Amomyrtus luma presentaron la mayor densidad. Estas dos últimas especies se desarrollaron bajo la cobertura de Chusquea macrostachya y Lophosoria quadripinnata. Drimys winteri y Embothrium coccineum se establecieron inmediatamente luego del disturbio antrópico de tala rasa y quema, confirmando el carácter pionero de ambas especies luego de disturbios de gran severidad. La regeneración de Nothofagus nitida fue escasa en el área, probablemente asociada a la baja disponibilidad y capacidad de dispersión de semillas desde bosques aledaños. La comprensión de la respuesta temprana de los bosques siempreverdes afectados por disturbios antrópicos es de gran importancia para asistir y guiar la restauración ecológica de estos ecosistemas forestales.

Palabras clave: bosque siempreverde, tala rasa, quema, diversidad florística.

\section{INTRODUCCIÓN}

$\mathrm{Al}$ sur de los $38^{\circ} \mathrm{S}$ en Chile, la Cordillera de la Costa presenta bosques templados siempreverdes poseedores de un alto número de endemismos y riqueza específica, condición que le ha permitido ser incluida dentro las 25 zonas prioritarias para la conservación de la biodiversidad mundial (Myers et al. 2000). Sin embargo, estos bosques han sido históricamente afectados por disturbios antrópicos, con la finalidad de extraer madera y habilitar terrenos para uso agrícola, ganadero y forestal (Donoso et al. 1999). Estas acciones, que por lo general se iniciaban con talas rasas 
o quemas, produjeron el reemplazo completo de rodales, facilitando el inicio de nuevas cohortes y provocando cambios importantes en la estructura, composición y extensión específica de estos bosques (Lara et al. 1997).

En la Cordillera de la Costa de la región de Los Ríos se encuentra ubicada la Reserva Costera Valdiviana (39 $56^{\circ}$ ' - $73^{\circ} 40^{\prime}$ O), área protegida privada de 50.250 ha que se caracteriza por su rica diversidad biológica y cultural. Florísticamente, esta área reúne 344 especies de plantas vasculares, $80 \%$ de ellas nativas, de las que un 7,6 \% se encuentra con problemas de conservación (CEA 2005). Previo a la creación de la reserva, se llevó a cabo un programa de sustitución de bosque nativo del tipo forestal siempreverde por plantaciones de especies exóticas principalmente de Eucalyptus globulus Labill., Eucalyptus nitens (Dean et Maiden) Maiden y Eucalyptus delegatensis R.T. Baker, abarcando 3.200 ha aproximadamente (Farías y Tecklin 2003). A fines del siglo pasado se sustituyeron, mediante talas rasas y quemas, cerca de 1.600 ha de bosque nativo adulto para plantarlas con las especies señaladas, pero posteriormente estos terrenos fueron abandonados. No existen estudios sobre la respuesta de la vegetación natural a los disturbios antrópicos señalados.

En un área alterada por fuego, las etapas de la sucesión se inician con el establecimiento de especies pioneras, entre las que destacan las formas de vida hemicriptófitas y terófitas (Grigera et al. 1996, Vidal y Reif 2011). En las primeras etapas de la sucesión y dependiendo del tipo de bosque, disturbio (y su severidad) y la historia de vida de las especies (Veblen et al. 2004), la diversidad de la flora y de la vegetación responderá gradual o discretamente a los gradientes y cambios drásticos en los factores del medioambiente, por lo que resulta imprescindible censar las especies que regeneran para comprender sus propiedades ecológicas y determinar las posibilidades que estas tienen de acceder al área, establecerse y competir (Donoso 1989a). Las respuestas ecológicas a los disturbios son específicas para cada sitio y muy relacionadas a los rasgos de vidas de las especies dominantes, al clima y en ocasiones a la acción animal doméstica (Veblen et al. 2004).

La literatura científica reporta diversos estudios sobre la flora y la vegetación en la Cordillera de la Costa, los que en su mayoría han sido llevados a cabo en bosques adultos del tipo forestal siempreverde o en bosques remanentes de coníferas luego de alteraciones antrópicas (Donoso 1989ab, Ramírez y San Martín 2005, Soto et al. 2010, Szejner et al. 2011), y donde especies arbóreas de la familia Myrtaceae y ejemplares de Embothrium coccineum J.R. et G. Forster y Drimys winteri J.R. Forst. et G. Forst., aparecen como las principales especies pioneras (Ramírez y San Martin 2005).

El objetivo de este estudio es comprender la recuperación natural del bosque siempreverde afectado por tala rasa, quema y abandono hace 14 años, en un sitio de 3,5 hectáreas. Para ello se planteó a) identificar las especies vasculares, b) caracterizar estructuralmente el área y c) determinar el patrón de establecimiento temporal de las principales especies arbóreas presentes. La hipótesis de trabajo presupone que la diversidad florística es mayoritariamente nativa, con una importante presencia de hemicriptófitas, y con las especies arbóreas Embothrium coccineum y Drimys winteri como especies pioneras post-disturbio antrópico.

\section{MÉTODOS}

Área de estudio. El área de estudio se ubica en la Reserva Costera Valdiviana (figura 1). Esta presenta clima oceánico con influencia mediterránea, con precipitaciones anuales entre $2.350 \mathrm{~mm}$ en las zonas bajas, hasta $4.000 \mathrm{~mm}$ en las zonas más altas, con una temperatura promedio anual de $12,0^{\circ} \mathrm{C}$ (Di Castri y Hajek 1976). Los suelos en los que se emplaza la Reserva Costera Valdiviana pertenecen a las series Hueicolla y La Pelada, caracterizadas por ser de origen metamórfico, derivados de micaesquistos, con grandes variaciones de espesor (moderadamente profundos a muy delgados), de textura arcillosa, fuertemente ácidos y susceptibles a erosión (CIREN 2003).

Para este estudio se seleccionó un área de 3,5 ha que había sido sometida a tala rasa y quema de desechos hace 14 años aproximadamente con la finalidad de plantar Eucalyptus spp. Sin embargo, luego de la quema, el terreno fue abandonado. Esta área colinda con un bosque siempreverde, caracterizado por Nothofagus nitida (Phil.) Krasser, Drimys winteri, Saxegothaea conspicua Lindl., Podocarpus nubigenus Lindl. y varias especies de Myrtaceae (1.880 árboles ha ${ }^{-1}, 83 \mathrm{~m}^{2} \mathrm{ha}^{-1}$ de área basal, 150 años aproximadamente $)^{1}$, y una plantación de E. globulus (800 árboles ha-1, $12 \mathrm{~m}^{2}$ ha $^{-1}$ de área basal y 13 años) ${ }^{2}$ (figura 1).

El área de estudio ocupa terrenos intermedios ondulados a quebrados (270 a $310 \mathrm{~m}$ s.n.m., ladera media y ladera alta respectivamente), con colinas de laderas convexas de 0 a $25 \%$ de pendiente con una exposición Este predominante. El drenaje externo del suelo es principalmente rápido. Según la descripción de dos perfiles de suelo y considerando la metodología de reconocimiento de Schlatter et al. (2003) y protocolos de análisis de Sadzawka et al. (2006), se observan suelos delgados, con profundidad de desarrollo moderado, con bajos nivel de pedregosidad en los primeros horizontes, densidad aparente y resistencia a la penetración media a alta, de moderado contenido de materia orgánica, muy ácidos, con bajos contenidos de bases, nitrógeno y fósforo, pero con altos niveles de aluminio. Texturas franco limosas a franco arenosas y estructuras redondeadas a masivas. El color de los suelos responde al matiz 10YR en todos los casos, existiendo algunas variaciones en la saturación y la intensidad, pero predominando el color amarillento oscuro. El drenaje interno es moderado a lento a mayor profundidad, con bajo contenido de agua aprovechable. La riqueza nutritiva principalmente se

Ortega-Jiménez S, ME González, A Lara, 2011. LIV Reunión Anual Sociedad de Biología de Chile y XVIII Reunión Anual Sociedad de Ecología de Chile.

Mario Romero-Mieres. Datos no publicados. 


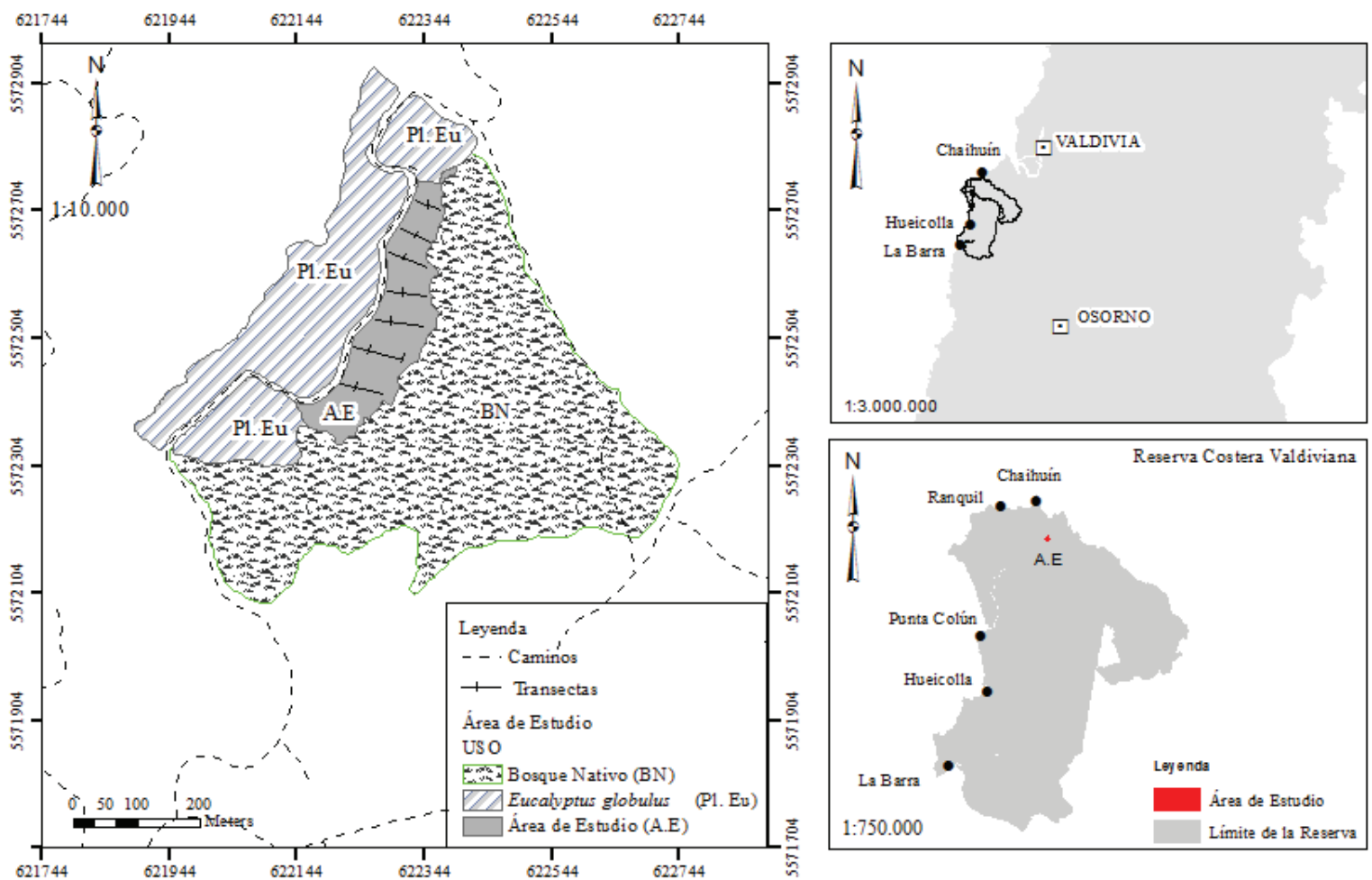

Figura 1. Ubicación de la Reserva Costera Valdiviana y área de estudio.

Location of the Valdivian Coastal Reserve and study area.

encuentra entre los primeros 3 a $4 \mathrm{~cm}$. El arraigamiento en ambos perfiles se concentró principalmente en los horizontes A y BA (anexos 1 y 2). El área presenta presión por ganado vacuno, preferentemente en los sectores de borde aledaños al camino, presentando además huellas de madereo y dispersas canchas de acopio de trozas menores.

Muestreo de la vegetación. Se establecieron 27 parcelas circulares $\left(50,2 \mathrm{~m}^{2}\right)$, ubicadas en siete transectas de orientación Oeste-Este (dirección pendiente) (figura 1). Las transectas fueron distribuidas equidistantes a lo largo de la faja NorteSur. En cada transecta la distancia entre parcelas fue de 18 $\mathrm{m}$ desde sus centros. En cada parcela se registró la especie arbórea y se midió el DAP (diámetro a 1,3 m) de todos los ejemplares vivos o muertos $\geq 5 \mathrm{~cm}$ (adultos). En cada parcela se distribuyeron sistemáticamente ocho subparcelas de $1 \mathrm{~m}^{2}$ separada por $1 \mathrm{~m}$ entre ellas. En estas subparcelas se contaron e identificaron brinzales (altura $>2 \mathrm{~m}$ y DAP $<5 \mathrm{~cm}$ ) y plántulas (altura $<2 \mathrm{~m}$ ) por especie. La composición florística y la cobertura estimada de cada especie fue determinada mediante el método de Braun-Blanquet (1979) $(+=<1 \%$, $1=1-5 \%, 2=6-25 \%, 3=26-50 \%, 4=51-75 \%, 5=76-$ $100 \%)$. Para la determinación de las fechas de establecimiento de las principales especies arbóreas se colectaron al azar discos basales de individuos ubicados entre las transectas del área de estudio. Su procesamiento y análisis siguió la metodología propuesta por Stokes y Smiley (1996).
Análisis de datos. Con la finalidad de evaluar un eventual efecto sobre la composición florística del área, la cercanía de este con la plantación de Eucalyptus globulus y con el bosque siempreverde, se identificaron cuatro sectores de Oeste a Este, siendo el sector 1 el más cercano a la plantación de Eucalyptus globulus y el sector 4 el más cercano al bosque siempreverde.

Para el análisis de la composición florística y riqueza de especies, estas se clasificaron en seis formas de crecimiento: arbórea, arbustiva, trepadoras-rastreras, epífitas, helechos terrestres y herbáceas. La clasificación y nomenclatura de las especies, el origen fitogeográfico y las formas de vida de estas se basó en los trabajos de Zuloaga et al. (2008), Marticorena y Quezada (1985) y la clasificación de Raunkiaer (Ellenberg y Mueller-Dombois 1966), respectivamente. A partir del registro de cobertura y frecuencia de cada especie se determinó la frecuencia y cobertura relativa y el valor de importancia de cada especie (Wikum y Shanholtzer 1978). De acuerdo a Hauenstein et al. 1988 y González (2000) se determinó el grado de intervención antrópica. Estos autores utilizan el origen fitogeográfico y las formas de vida de las especies para establecer el grado de disturbio antrópico de un lugar, a través de la relación especies nativas versus introducidas.

La composición y estructura arbórea del área de estudio se caracterizó en términos de densidad y diámetro de sus especies. Para los distintos sectores identificados se comparó 
la densidad de plántulas y brinzales por especie mediante la prueba no paramétrica de Kruskall-Wallis $(P<0,05)$. Con las edades de los individuos vivos se determinó el patrón de establecimiento post-disturbio de las principales especies.

\section{RESULTADOS}

Caracterización florística. La flora vascular comprendió 77 especies, de las cuales 47 son angiospermas dicotiledóneas (61 \%), 20 angiospermas monocotiledóneas (26 \%), ocho pteridófitos $(10,4 \%)$ y dos gimnospermas $(2,6 \%)$ (cuadro 1).

El origen fitogeográfico muestra que son dominantes las especies nativas (84,4\%). El espectro biológico fue representado por 33 fanerófitos (43\%), 27 hemicriptófitos (35\%), 10 fanerófitos trepadores (13\%), tres caméfitos (4\%), dos terófitos (3\%), un epífito (1\%) y un geófito (1\%). Las especies se agrupan en un total de 36 familias y 59 géneros, siendo las más representativas en riqueza específica las familias Myrtaceae (9), Asteraceae (8) y Poaceae (6). El género que más representantes tiene es Blechnum L., con cuatro especies. Las especies que presentan mayor valor de importancia son Nertera granadensis (Mutis ex L.f.) Druce (30,5), Lophosoria quadripinnata (J.F. Gmel.) C. Chr. $(28,2)$, Leontodon saxatilis Lam. (16,3), Baccharis sphaerocephala H. et A. $(13,3)$ y Drimys winteri $(12,7)$ (cuadro 1).

Caracterización de la estructura. Según la forma de crecimiento, se identificaron 19 especies arbóreas $(24,7 \%), 15$ arbustivas (19,5\%), 23 herbáceas (29,9 \%), 12 trepadoras/ rastreras $(15,6 \%)$, siete helechos terrestres $(9,1 \%)$ y un hele- cho epífito $(1,3 \%)$. Destacan en estas formas de crecimiento, según su valor de importancia, las especies Drimys winteri, Baccharis sphaerocephala, Leontodon saxatilis, Nertera granadensis, Blechnum cordatum (Desv.) Hieron e Hymenophyllum pectinatum Cav., respectivamente (cuadro 1).

El número de plántulas registradas en el área fue de 20.787 ejemplares ha ${ }^{-1}$, siendo D. winteri, Saxegothaea conspicua y Amomyrtus luma (Mol.) Legr. et Kaus. las especies más numerosas (cuadro 2). El número de brinzales registrado fue de 12.593 ejemplares ha ${ }^{-1}$, con Drimys winteri, Amomyrtus luma y Saxegothaea conspicua como las especies mayoritarias. Asimismo, el número de ejemplares con DAP $\geq 5 \mathrm{~cm}$ (adultos) registrados fue de 116 árboles $\mathrm{ha}^{-1}$, donde nuevamente Drimys winteri, Amomyrtus luma y Saxegothaea conspicua fueron las especies más numerosas (cuadro 2). La presencia de Nothofagus nitida en el área fue reducida respecto de las demás especies arbóreas y sólo se registró en los sectores más cercanos al bosque siempreverde colindante (sector 4) (cuadro 2). Solo se observaron diferencias estadísticas significativas entre el número de plántulas presentes entre los sectores analizados (cuadro 3). En aquellas zonas colindantes con el bosque siempreverde (más húmedas), las especies dominantes son aquellas de forma arbustiva, como Baccharis sphaerocephala, Greigia sphacelata (R. et P.) Regel y Chusquea montana Phil. f. nigricans (Phil.) Matthei.

El sector de estudio presenta individuos con rango de edades de 7 (año 2006) a 18 años (año 1995). Drimys winteri, Amomyrtus luma y Podocarpus nubigenus presentan algunos ejemplares que son parte de las especies que se encontraban antes del disturbio (figura 2).

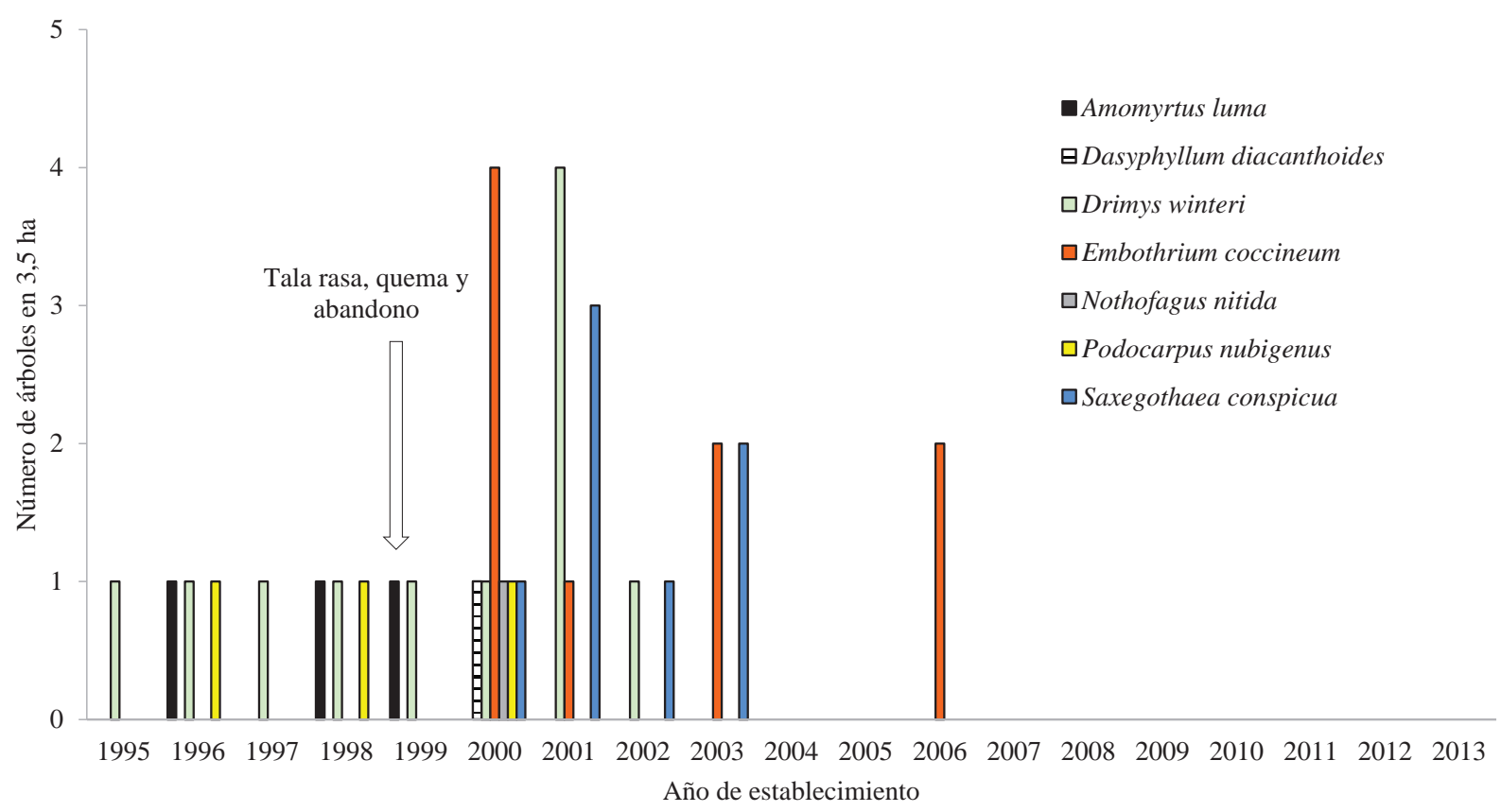

Figura 2. Año de establecimiento de las principales especies arbóreas del área de estudio. Year of establishment of main tree species in the study area. 
Cuadro 1. Composición florística, forma de vida, forma de crecimiento y valor de importancia de las especies vasculares en el área de estudio.

Floristic composition, life form, growth form and importance value of vascular species in the study area.

\begin{tabular}{|c|c|c|c|c|c|c|c|c|}
\hline Nombre científico & Nombre común & Familia & $\mathrm{OF}$ & FV & FC & Fr & $\mathrm{Cr}$ & VI \\
\hline \multicolumn{9}{|l|}{ PTERIDOPHYTA } \\
\hline Blechnum cordatum (Desv.) Hieron. & Costilla de vaca & Blechnaceae & $\mathrm{N}$ & $\mathrm{H}$ & Ht & 4,30 & 5,28 & 9,6 \\
\hline Blechnum hastatum Kaulf. & Palmilla & Blechnaceae & $\mathrm{N}$ & $\mathrm{H}$ & Ht & 0,07 & 0,05 & 0,1 \\
\hline Blechnum magellanicum (Desv.) Mett. & Katalapi & Blechnaceae & $\mathrm{N}$ & $\mathrm{H}$ & Ht & 0,13 & 0,07 & 0,2 \\
\hline Blechnum mochaenum G. Kunkel & Iquide & Blechnaceae & $\mathrm{N}$ & $\mathrm{H}$ & Ht & 0,07 & 0,00 & 0,1 \\
\hline Blechnum penna-marina (Poir.) Kuhn & Punke & Blechnaceae & $\mathrm{N}$ & $\mathrm{H}$ & $\mathrm{Ht}$ & 0,67 & 1,19 & 1,9 \\
\hline Hymenophyllum pectinatum Cav. & Helecho película & Hymenophyllaceae & $\mathrm{N}$ & $\mathrm{E}$ & $\mathrm{E}$ & 0,07 & 0,01 & 0,1 \\
\hline Lophosoria quadripinnata (J.F. Gmel.) C. Chr. & Ampe & Dicksoniaceae & $\mathrm{N}$ & $\mathrm{H}$ & $\mathrm{Ht}$ & 7,52 & 20,72 & 28,2 \\
\hline Megalastrum spectabile (Kaulf.) A.R. Sm. et R.C. Moran & Pesebre & Drypteridaceae & $\mathrm{N}$ & $\mathrm{H}$ & Ht & 0,07 & 0,05 & 0,1 \\
\hline \multicolumn{9}{|l|}{ GYMNOSPERMAE } \\
\hline Podocarpus nubigenus Lindl. & Mañío macho & Podocarpaceae & $\mathrm{N}$ & $\mathrm{F}$ & A & 0,34 & 0,23 & 0,6 \\
\hline Saxegothaea conspicua Lindl. & Mañío hembra & Podocarpaceae & $\mathrm{N}$ & $\mathrm{F}$ & A & 4,03 & 2,11 & 6,1 \\
\hline \multicolumn{9}{|l|}{ ANGIOSPERMAE (DICOTYLEDONEAE) } \\
\hline Amomyrtus luma (Molina) D. Legrand et Kausel & Luma & Myrtaceae & $\mathrm{N}$ & F & A & 2,28 & 1,84 & 4,1 \\
\hline Amomyrtus meli (Phil.) D. Legrand et Kausel & Meli & Myrtaceae & $\mathrm{N}$ & F & A & 0,60 & 0,18 & 0,8 \\
\hline Asteranthera ovata (Cav.) Hanst. & Estrellita del bosque & Gesneriaceae & $\mathrm{N}$ & $\mathrm{Ft}$ & $\mathrm{T} / \mathrm{R}$ & 0,27 & 0,09 & 0,4 \\
\hline Azara lanceolata Hook. f. & Aromo de castilla & Salicaceae & $\mathrm{N}$ & $\mathrm{F}$ & $\mathrm{Ar}$ & 0,07 & 0,01 & 0,1 \\
\hline Baccharis sphaerocephala Hook. et Arn. & Radín & Asteraceae & $\mathrm{N}$ & $\mathrm{F}$ & $\mathrm{Ar}$ & 6,25 & 7,06 & 13,3 \\
\hline Baccharis obovata Hook. et Arn. & Chilca & Asteraceae & $\mathrm{N}$ & $\mathrm{F}$ & $\mathrm{Ar}$ & 2,08 & 0,96 & 3,0 \\
\hline Caldcluvia paniculata (Cav.) D. Don & Tiaca & Cunoniaceae & $\mathrm{N}$ & $\mathrm{F}$ & A & 0,07 & 0,00 & 0,1 \\
\hline Campsidium valdivianum (Phil.) Skottsb. & Voqui & Bignoniaceae & $\mathrm{N}$ & $\mathrm{Ft}$ & $T / R$ & 2,75 & 0,88 & 3,6 \\
\hline Dasyphyllum diacanthoides (Less.) Cabrera & Trevo & Asteraceae & $\mathrm{N}$ & $\mathrm{F}$ & A & 0,07 & 0,13 & 0,2 \\
\hline Drimys winteri J.R.Forst. et G.Forst. & Canelo & Winteraceae & $\mathrm{N}$ & $\mathrm{F}$ & A & 5,98 & 6,69 & 12,7 \\
\hline Elytropus chilensis (A.DC.) Müll. Arg. & Quilmay & Apocynaceae & $\mathrm{N}$ & $\mathrm{Ft}$ & $\mathrm{T} / \mathrm{R}$ & 0,07 & 0,00 & 0,1 \\
\hline Embothrium coccineum J.R. Forst. et G. Forst. & Notro & Proteaceae & $\mathrm{N}$ & $\mathrm{F}$ & A & 0,74 & 0,81 & 1,5 \\
\hline Eucryphia cordifolia Cav. & Ulmo & Eucryphiaceae & $\mathrm{N}$ & $\mathrm{F}$ & A & 0,20 & 0,06 & 0,3 \\
\hline Galium hypocarpium (L.) Endl. ex Griseb. & Relbún & Rubiaceae & $\mathrm{N}$ & $\mathrm{C}$ & $\mathrm{T} / \mathrm{R}$ & 0,54 & 0,03 & 0,6 \\
\hline Gamochaeta coarctata (Willd.) Kerguélen & Nafalium & Asteraceae & I & $\mathrm{H}$ & $\mathrm{H}$ & 0,47 & 0,01 & 0,5 \\
\hline Gaultheria insana (Molina) D.J. Middlenton & Chaura & Ericaceae & $\mathrm{N}$ & $\mathrm{F}$ & $\mathrm{Ar}$ & 0,07 & 0,01 & 0,1 \\
\hline Gaultheria phillyreifolia (Pers.) Sleumer & Chaura & Ericaceae & $\mathrm{N}$ & $\mathrm{F}$ & $\mathrm{Ar}$ & 0,40 & 0,07 & 0,5 \\
\hline Gevuina avellana Mol. & Avellano & Proteaceae & $\mathrm{N}$ & $\mathrm{F}$ & A & 0,47 & 0,08 & 0,6 \\
\hline Griselinia jodinifolia (Griseb.) Taub. & Yelmo & Griseliniaceae & $\mathrm{N}$ & $\mathrm{F}$ & $\mathrm{Ar}$ & 0,13 & 0,14 & 0,3 \\
\hline Griselinia ruscifolia (Clos) Ball & Lilinquén & Griseliniaceae & $\mathrm{N}$ & $\mathrm{Ft}$ & $\mathrm{T} / \mathrm{R}$ & 0,13 & 0,01 & 0,1 \\
\hline Hydrocotyle chamaemorus Cham. et Schltdl. & Malva del monte & Apiaceae & $\mathrm{N}$ & $\mathrm{H}$ & $\mathrm{H}$ & 0,07 & 0,00 & 0,1 \\
\hline Hypochaeris radicata $\mathrm{L}$. & Hierba del chancho & Asteraceae & I & $\mathrm{H}$ & $\mathrm{H}$ & 4,16 & 3,66 & 7,8 \\
\hline Laureliopsis philippiana (Looser) Schodde & Тера & Monimiaceae & $\mathrm{N}$ & $\mathrm{F}$ & A & 0,47 & 0,03 & 0,5 \\
\hline Leontodon saxatilis Lam. & Chinilla & Asteraceae & I & $\mathrm{H}$ & $\mathrm{H}$ & 8,26 & 8,03 & 16,3 \\
\hline Lomatia ferruginea (Cav.) R.Br. & Fuinque & Proteaceae & $\mathrm{N}$ & $\mathrm{F}$ & A & 0,13 & 0,06 & 0,2 \\
\hline Lotus pedunculatus Cav. & Alfalfa chilota & Fabaceae & I & $\mathrm{H}$ & $\mathrm{H}$ & 0,27 & 0,07 & 0,3 \\
\hline Luma apiculata (DC.) Burret & Arrayán & Myrtaceae & $\mathrm{N}$ & $\mathrm{F}$ & A & 0,07 & 0,01 & 0,1 \\
\hline
\end{tabular}


Continuación cuadro 1

\begin{tabular}{|c|c|c|c|c|c|c|c|c|}
\hline Mitraria coccinea Cav. & Botellita & Gesneriaceae & $\mathrm{N}$ & $\mathrm{Ft}$ & $\mathrm{T} / \mathrm{R}$ & 1,88 & 2,65 & 4,5 \\
\hline Muehlenbeckia hastulata (Sm.) I.M. Johnst. & Quilo & Polygonaceae & $\mathrm{N}$ & $\mathrm{Ft}$ & $\mathrm{T} / \mathrm{R}$ & 0,60 & 0,24 & 0,8 \\
\hline Myrceugenia ovata (Hook. et Arn.) O. Berg & Patagüilla & Myrtaceae & $\mathrm{N}$ & F & A & 1,21 & 0,48 & 1,7 \\
\hline Myrceugenia parvifolia (DC.) Kausel & Chilchilco & Myrtaceae & $\mathrm{N}$ & F & A & 0,40 & 0,08 & 0,5 \\
\hline Myrceugenia planipes (Hook. et Arn.) O. Berg & Patagua de Valdivia & Myrtaceae & $\mathrm{N}$ & F & A & 0,34 & 0,07 & 0,4 \\
\hline Nertera granadensis (Mutis ex L.f.) Druce & Coralito & Rubiaceae & $\mathrm{N}$ & $\mathrm{H}$ & $\mathrm{T} / \mathrm{R}$ & 12,22 & 18,27 & 30,5 \\
\hline Nothofagus nitida (Phil.) Krasser & Coigüe de Chiloé & Fagaceae & $\mathrm{N}$ & F & A & 0,47 & 0,68 & 1,2 \\
\hline Ovidia andina (Poepp. et Endl.) Meisn. & Pillo-pillo & Thymelaeaceae & $\mathrm{N}$ & F & A & 0,40 & 0,07 & 0,5 \\
\hline Plantago lanceolata $\mathrm{L}$. & Siete venas & Plantaginaceae & I & $\mathrm{H}$ & $\mathrm{H}$ & 0,13 & 0,02 & 0,2 \\
\hline Prunella vulgaris L. & Hierba mora & Lamiaceae & I & $\mathrm{C}$ & $\mathrm{H}$ & 0,27 & 0,08 & 0,3 \\
\hline Raukaua laetevirens (Gay) Frodin & Sauco del diablo & Araliaceae & $\mathrm{N}$ & F & $\mathrm{Ar}$ & 0,07 & 0,00 & 0,1 \\
\hline Rubus constrictus P.J. Müll. et Lefèvre & Zarzamora & Rosaceae & I & F & $\mathrm{Ar}$ & 0,40 & 0,04 & 0,4 \\
\hline Rumex acetocella L. & Vinagrillo & Polygonaceae & I & $\mathrm{H}$ & $\mathrm{H}$ & 0,13 & 0,06 & 0,2 \\
\hline Senecio otites Kunze ex DC. & Trompetilla & Asteraceae & $\mathrm{N}$ & $\mathrm{H}$ & $\mathrm{H}$ & 0,74 & 0,11 & 0,8 \\
\hline Taraxacum officinale G. Weber ex F.H. Wigg. & Diente de león & Asteraceae & I & $\mathrm{H}$ & $\mathrm{H}$ & 0,81 & 0,11 & 0,9 \\
\hline Tepualia stipularis (Hook. et Arn.) Griseb. & Tepú & Myrtaceae & $\mathrm{N}$ & F & A & 0,87 & 0,47 & 1,3 \\
\hline Ugni candollei (Barnéoud) O. Berg & Murta blanca & Myrtaceae & $\mathrm{N}$ & F & $\mathrm{Ar}$ & 0,07 & 0,05 & 0,1 \\
\hline Ugni molinae Turcz. & Murta & Myrtaceae & $\mathrm{N}$ & F & $\mathrm{Ar}$ & 1,21 & 0,48 & 1,7 \\
\hline Ulex europaeus L. & Pica-pica & Fabaceae & I & F & $\mathrm{Ar}$ & 0,07 & 0,01 & 0,1 \\
\hline Viola reichei Skottsb. & Violeta & Violaceae & $\mathrm{N}$ & $\mathrm{C}$ & $\mathrm{H}$ & 1,41 & 1,74 & 3,2 \\
\hline \multicolumn{9}{|l|}{ ANGIOSPERMAE (MONOCOTYLEDONEAE) } \\
\hline Agrostis capillaris L. & Chépica & Poaceae & I & $\mathrm{H}$ & $\mathrm{H}$ & 3,02 & 1,10 & 4,1 \\
\hline Carex fuscula d'Urv. & Cortadera & Cyperaceae & $\mathrm{N}$ & $\mathrm{H}$ & $\mathrm{H}$ & 4,03 & 2,25 & 6,3 \\
\hline Carex chilensis Brongn. ex Duperrey & Cortadera & Cyperaceae & $\mathrm{N}$ & $\mathrm{H}$ & $\mathrm{H}$ & 0,20 & 0,03 & 0,2 \\
\hline Chusquea macrostachya Phil. & Quila & Poaceae & $\mathrm{N}$ & $\mathrm{F}$ & $\mathrm{Ar}$ & 1,41 & 2,89 & 4,3 \\
\hline Chusquea montana Phil. f. nigricans (Phil.) Matthei & Quila enana & Poaceae & $\mathrm{N}$ & $\mathrm{F}$ & Ar & 0,74 & 1,65 & 2,4 \\
\hline Chusquea uliginosa Phil. & Quila & Poaceae & $\mathrm{N}$ & $\mathrm{F}$ & $\mathrm{Ar}$ & 0,47 & 0,74 & 1,2 \\
\hline Gavilea littoralis (Phil.) M.N.Correa & Orquídea & Orchidaceae & $\mathrm{N}$ & G & $\mathrm{H}$ & 0,67 & 0,26 & 0,9 \\
\hline Greigia sphacelata (R. et P.) Regel & Chupón & Bromeliaceae & $\mathrm{N}$ & $\mathrm{H}$ & $\mathrm{Ar}$ & 0,74 & 0,65 & 1,4 \\
\hline Holcus lanatus L. & Pasto miel & Poaceae & I & $\mathrm{T}$ & $\mathrm{H}$ & 0,34 & 0,08 & 0,4 \\
\hline Juncus pallescens Lam. & Junco & Juncaceae & $\mathrm{N}$ & $\mathrm{H}$ & $\mathrm{H}$ & 0,47 & 0,05 & 0,5 \\
\hline Juncus planifolius R. Br. & Junquillo pasto & Juncaceae & $\mathrm{N}$ & $\mathrm{T}$ & $\mathrm{H}$ & 0,40 & 0,28 & 0,7 \\
\hline Juncus procerus E. Mey. & Junco & Juncaceae & $\mathrm{N}$ & $\mathrm{H}$ & $\mathrm{H}$ & 0,54 & 0,35 & 0,9 \\
\hline Lapageria rosea Ruiz et Pav. & Copihue & Philesiaceae & $\mathrm{N}$ & $\mathrm{Ft}$ & $\mathrm{T} / \mathrm{R}$ & 5,04 & 1,97 & 7,0 \\
\hline Luzuriaga polyphylla (Hook.) J.F. Macbr. & Quilineja & Luzuriagaceae & $\mathrm{N}$ & $\mathrm{Ft}$ & $\mathrm{T} / \mathrm{R}$ & 1,14 & 0,19 & 1,3 \\
\hline Luzuriaga radicans Ruiz et Pav. & Quilineja & Luzuriagaceae & $\mathrm{N}$ & Ft & $\mathrm{T} / \mathrm{R}$ & 0,20 & 0,07 & 0,3 \\
\hline Philesia magellanica J.F. Gmel. & Coicopihue & Philesiaceae & $\mathrm{N}$ & $\mathrm{Ft}$ & $\mathrm{T} / \mathrm{R}$ & 0,27 & 0,07 & 0,3 \\
\hline Polypogon australis Brongn. & Cola de ratón & Poaceae & $\mathrm{N}$ & $\mathrm{H}$ & $\mathrm{H}$ & 0,20 & 0,02 & 0,2 \\
\hline Scirpus inundatus (R. Br.) Poir. & Chan-chán & Cyperaceae & $\mathrm{N}$ & $\mathrm{H}$ & $\mathrm{H}$ & 0,07 & 0,01 & 0,1 \\
\hline Uncinia multifaria Nees ex Boott & Cortadera & Cyperaceae & $\mathrm{N}$ & $\mathrm{H}$ & $\mathrm{H}$ & 1,34 & 0,73 & 2,1 \\
\hline Uncinia tenuis Poepp. ex Kunth & Cortadera & Cyperaceae & $\mathrm{N}$ & $\mathrm{H}$ & $\mathrm{H}$ & 0,81 & 0,36 & 1,2 \\
\hline
\end{tabular}

$\mathrm{OF}=$ origen fitogeográfico $(\mathrm{N}=$ nativo, $\mathrm{I}=$ introducido $), \mathrm{FV}=$ forma de vida $(\mathrm{F}=$ fanerófito, $\mathrm{Ft}=$ fanerófito trepador, $\mathrm{H}=$ hemicriptófito, $\mathrm{C}=$ caméfito, $\mathrm{G}=$ geófito, $\mathrm{T}=$ terófito, $\mathrm{E}=$ epífito $), \mathrm{FC}=$ forma de crecimiento $(\mathrm{A}=$ arbóreo, $\mathrm{Ar}=$ arbustivo, $\mathrm{T} / \mathrm{R}=$ trepadoras/rastreras, $\mathrm{E}=\mathrm{epífitas}, \mathrm{Ht}=$ helechos terrestres, $\mathrm{H}$ = herbáceas, $\mathrm{Fr}=$ frecuencia relativa, $\mathrm{Cr}=$ cobertura relativa, $\mathrm{VI}=$ valor de importancia. 
Cuadro 2. Densidad y estructura diamétrica por especie arbórea $\left(\mathrm{N}^{\mathrm{o}} \mathrm{ha}^{-1}\right)$.

Density and diametric structure of tree species $\left(\mathrm{N} \mathrm{ha}^{-1}\right)$.

\begin{tabular}{|c|c|c|c|c|c|c|c|c|c|c|c|c|c|c|c|c|c|}
\hline \multirow{2}{*}{ Especie } & \multirow{2}{*}{$\begin{array}{c}\text { Total } \\
\mathrm{p}\end{array}$} & \multirow{2}{*}{$\begin{array}{c}\text { Total } \\
\mathrm{b}\end{array}$} & \multicolumn{3}{|c|}{$\begin{array}{l}\text { Total adultos } \\
\text { por cd }(\mathrm{cm})\end{array}$} & \multicolumn{3}{|c|}{ Sector 1} & \multicolumn{3}{|c|}{ Sector 2} & \multicolumn{3}{|c|}{ Sector 3} & \multicolumn{3}{|c|}{ Sector 4} \\
\hline & & & 10 & 15 & $\mathrm{a}$ & $\mathrm{p}$ & $\mathrm{b}$ & a & $\mathrm{p}$ & $\mathrm{b}$ & a & $\mathrm{p}$ & $\mathrm{b}$ & $\mathrm{a}$ & $\mathrm{p}$ & $\mathrm{b}$ & a \\
\hline A. luma & 1.481 & 2.130 & 29 & 0 & 29 & 139 & 139 & 0 & 232 & 1.250 & 29 & 833 & 556 & 0 & 278 & 185 & 0 \\
\hline A. meli & 93 & 46 & 0 & 0 & 0 & 0 & 0 & 0 & 0 & 0 & 0 & 46 & 0 & 0 & 46 & 46 & 0 \\
\hline D. diacanthoides & 0 & 0 & 7 & 0 & 7 & 0 & 0 & 0 & 0 & 0 & 0 & 0 & 0 & 7 & 0 & 0 & 0 \\
\hline D. winteri & 12.639 & 7.083 & 44 & 7 & 51 & 1.296 & 880 & 7 & 2.037 & 2.546 & 22 & 4.861 & 2.269 & 22 & 4.444 & 1.389 & 0 \\
\hline E. coccineum & 185 & 231 & 7 & 0 & 7 & 46 & 0 & 0 & 0 & 0 & 0 & 139 & 139 & 7 & 0 & 93 & 0 \\
\hline E. cordifolia & 185 & 93 & 0 & 0 & 0 & 46 & 0 & 0 & 0 & 46 & 0 & 139 & 0 & 0 & 0 & 46 & 0 \\
\hline G. avellana & 417 & 139 & 0 & 0 & 0 & 0 & 0 & 0 & 0 & 0 & 0 & 139 & 0 & 0 & 278 & 139 & 0 \\
\hline L. philippiana & 324 & 46 & 0 & 0 & 0 & 0 & 0 & 0 & 0 & 46 & 0 & 0 & 0 & 0 & 324 & 0 & 0 \\
\hline L. ferruginea & 93 & 46 & 0 & 0 & 0 & 0 & 0 & 0 & 0 & 0 & 0 & 0 & 0 & 0 & 93 & 46 & 0 \\
\hline L. apiculata & 46 & 0 & 0 & 0 & 0 & 46 & 0 & 0 & 0 & 0 & 0 & 0 & 0 & 0 & 0 & 0 & 0 \\
\hline M. ovata & 231 & 231 & 0 & 0 & 0 & 0 & 46 & 0 & 139 & 46 & 0 & 46 & 93 & 0 & 46 & 46 & 0 \\
\hline M. parvifolia & 231 & 0 & 0 & 0 & 0 & 0 & 0 & 0 & 0 & 0 & 0 & 0 & 0 & 0 & 232 & 0 & 0 \\
\hline M. planipes & 185 & 46 & 0 & 0 & 0 & 0 & 46 & 0 & 46 & 0 & 0 & 139 & 0 & 0 & 0 & 0 & 0 \\
\hline N. nitida & 185 & 93 & 7 & 0 & 7 & 0 & 0 & 0 & 0 & 0 & 0 & 46 & 0 & 7 & 139 & 93 & 0 \\
\hline O. andina & 93 & 0 & 0 & 0 & 0 & 0 & 0 & 0 & 0 & 0 & 0 & 0 & 0 & 0 & 93 & 0 & 0 \\
\hline P. nubigenus & 278 & 46 & 0 & 0 & 0 & 46 & 0 & 0 & 0 & 0 & 0 & 93 & 0 & 0 & 139 & 46 & 0 \\
\hline S. conspicua & 3.565 & 1.759 & 15 & 0 & 15 & 232 & 232 & 0 & 324 & 741 & 0 & 1.528 & 509 & 0 & 1.481 & 278 & 15 \\
\hline T. stipularis & 556 & 602 & 0 & 0 & 0 & 278 & 0 & 0 & 0 & 0 & 0 & 278 & 602 & 0 & 0 & 0 & 0 \\
\hline Total & 20.787 & 12.593 & 109 & 7 & 116 & 2.129 & 1.343 & 7 & 2.778 & 4.675 & 51 & 8.287 & 4.168 & 43 & 7.593 & 2.407 & 15 \\
\hline
\end{tabular}

a= árboles, b= brinzales y p= plántulas, cd= clases diamétricas.

Cuadro 3. Prueba de Kruskal-Wallis para densidad de plántulas, brinzales y adultos entre sectores $(1,2,3,4)$.

Kruskal-Wallis test for tree regeneration between sectors (1, $2,3,4)$.

\begin{tabular}{lccc}
\hline \multicolumn{1}{c}{ Parámetro } & Plántulas & Brinzales & Adultos \\
\hline H (valor observado) & $39,419 *$ & 23,694 & 6,657 \\
H (valor crítico) & 38,885 & 38,885 & 38,885 \\
GDL & 26 & 26 & 26 \\
$P$-valor unilateral & 0,044 & 0,593 & 1,000 \\
Alfa & 0,05 & 0,05 & 0,05 \\
\hline
\end{tabular}

(*): diferencia estadísticamente significativa.

\section{DISCUSIÓN}

Composición florística. El origen fitogeográfico indica un $15,6 \%$ de especies introducidas, lo que sumado a la presencia de hemicriptófitos exóticos, entre los que destacan por valor de importancia Agrostis capillaris L., Hypochaeris radicata L. y Leontodon saxatilis, dan cuenta de inter- vención antrópica en el sector (cuadro 1) (Hauenstein et al. 1988, González 2000). Esto también se manifiesta al observar una alta densidad aparente y resistencia a la penetración del suelo en el área de estudio (anexo 1), similares a los encontrados en terrenos de cultivo (Honorato 2000).

La forma de vida hemicriptófíta sigue al hombre en el proceso de culturización de los ecosistemas y estaría ligada, al igual que terófitos y geófitos, a condiciones de sequía, especialmente en época estival (Grigera et al. 1996). Si bien la forma de vida predominante es la fanerófita (cuadro 1), vinculada a las altas tasas pluviométricas del área de estudio, en verano, esta se deseca fácilmente, sobre todo en el sector más cercano al camino y más plano, mientras que en invierno se satura. El desarrollo in situ del suelo sobre material metamórfico de tipo micaesquistos facilita este desecamiento (Donoso et al. 1999).

La cercanía del área con caminos forestales, plantación de E. globulus y tránsito permanente de animales (principalmente bovinos) facilitan la llegada y permanencia de hemicriptófitas introducidas de rápido crecimiento y muy invasivas, como Gamochaeta coarctata (Willd.) Kerguélen, Taraxacum officinale G. Weber ex F.H. Wigg., Lotus pedunculatus Cav., Plantago lanceolata L., Rumex ace- 
tocella L. y Holcus lanatus L. Del mismo modo, estas condiciones facilitan la llegada al área de dos especies arbustivas introducidas de gran capacidad reproductiva y regenerativa: Rubus constrictus P.J. Müll. et Lefèvre y Ulex europaeus L. (Weinberger 1997, Muñoz 2009). Sin embargo, ambas especies presentan baja cobertura (cuadro 1) y se hallan principalmente hacia el borde del camino o en los lugares donde la cobertura de otras especies arbóreas y arbustivas es baja. Esta baja cobertura hace que no constituyan una amenaza actual para el establecimiento de las especies nativas.

Si bien la presencia de animales domésticos constituye un agente causal de impacto sobre todas las especies del área de estudio, es importante destacar el impacto que ocasionan sobre especies de baja frecuencia relativa, como se observa en la orquídea geófita nativa presente en el área, Gavilea littoralis (Phil.) M. N. Correa, cuyos ejemplares presentan signos notorios de "ramoneo" por animales bovinos.

Estructura y patrón de establecimiento. Considerando las formas de crecimiento, se observa diferencias de composición, dadas principalmente por variables topográficas. En sectores de ladera alta (cercano al camino), la vegetación es principalmente herbácea-arbustiva, predominando Uncinia spp., Carex spp., Juncus spp., Baccharis spp. y Lophosoria quadripinnata, con escasos representantes arbóreos de mirtáceas y ejemplares de Drimys winteri, Podocarpus nubigenus y Saxegothaea conspicua. Mientras que en ladera media (cercano al bosque siempreverde), la vegetación es principalmente arborescente, siendo Drimys winteri y Saxegothaea conspicua las más abundantes, coexistiendo con Dasyphyllum diacanthoides (Less.) Cabrera, Gevuina avellana Mol., Myrceugenia planipes (Hook. et Arn.) O. Berg y Myrceugenia ovata (Hook. et Arn.) O. Berg. En este último sector, la cercanía de cursos de agua y la cobertura otorgada por Lophosoria quadripinnata y Chusquea spp., le confieren al sector condiciones óptimas para la regeneración de especies arbóreas de tolerancia media a muy tolerantes (Donoso 1989a, Donoso et al. 1999).

El área de estudio presenta a Drimys winteri y Embothrium coccineum como las especies arbóreas pioneras principales (figura 2), confirmando que estas especies ocupan tempranamente sitios alterados (Ramírez y San Martín 2005). Sin embargo, su presencia en términos de densidad (cuadro 2) es muy baja en comparación a lo informado por Donoso (1989a) para la Cordillera de la Costa.

Donoso (1989a) señala densidades de plántulas de Drimys winteri cercanas a 26.000 individuos por hectárea, posterior a tres años de aplicado el método de tala rasa en la Cordillera de la Costa. El número de ejemplares registrados en el presente estudio es muy inferior a esa densidad, lo que sugiere que las condiciones iniciales de sitio no fueron las óptimas para la especie. Donoso et al. (2006) informan que Drimys winteri regenera exitosamente cuando la humedad del suelo es permanente y de buen drenaje, sumada a una buena disponibilidad de materia or- gánica producida por troncos caídos y en estado avanzado de pudrición. Estas condiciones no se manifiestan en los sectores más expuestos y transitados (orilla de camino) del área estudiada, ya que en el verano el suelo se deseca rápidamente, sumado además al moderado contenido de materia orgánica existente en ellos y a la alta relación carbono/ nitrógeno (anexo 2), que dificulta la descomposición de la materia orgánica y la mineralización de nitrógeno, y en consecuencia, el crecimiento y desarrollo de las especies arbóreas allí presentes. Además, luego de la tala rasa esta área fue quemada, lo que pudo haber incidido fuertemente en la materia orgánica y material leñoso disponible, y en las condiciones físicas y químicas del suelo. Drimys winteri es una especie de tolerancia media (Donoso 1989a) y su regeneración se observó principalmente en las zonas más protegidas, húmedas y cercanas al bosque siempreverde colindante y donde las coberturas de Nertera granadensis, Chusquea macrostachya Phil. y Lophosoria quadripinnata fueron mayores.

Los ejemplares de Embothrium coccineum se observaron en condiciones de mayor intensidad de luz y con suelos más expuestos, demostrando su condición de especie muy intolerante a la sombra (Donoso 1989a) y pionera en situaciones de tala rasa, debido principalmente a la facilidad de dispersión por viento de sus semillas (Donoso et al. 1999) y a sus propiedades fisiológicas que le permiten adaptarse a diversos ambientes, sobre todo en sitios inhóspitos como laderas y áreas taladas (Alberdi y Donoso 2004).

En el caso de las mirtáceas, Donoso (1994) menciona valores que oscilan entre los 8.500 hasta 72.000 plántulas $\mathrm{ha}^{-1}$, mientras que en este estudio el número de plántulas no superó las 3.000 individuos ha-1 ${ }^{-1}$ En términos de plántulas, brinzales y adultos, Amomyrtus luma es la especie mejor representada y cuya presencia, principalmente bajo el follaje de Chusquea macrostachya y Lophosoria quadripinnata, sugiere un potencial efecto nodriza (Callaway 1995) de estas especies, y donde el suelo presenta mantillo húmedo, condición requerida para la germinación de sus semillas (Donoso et al. 1999). Sin embargo, en los sectores más expuestos, los brinzales y adultos de Amomyrtus luma, algunos agrupados, supone un modo de reproducción vegetativa, característico en esta especie de tolerancia media (Donoso 1989a). La presencia de Myrceugenia parvifolia (DC.) Kausel, Myrceugenia planipes у Tериаlia stipularis (Hook. et Arn.) Griseb. en los sectores con mayor cobertura y de condiciones de sitio más húmedos, confirma su condición de especies tolerantes a la sombra (Donoso 1989a), mientras que Myrceugenia ovata prácticamente solo se presenta en los sectores más soleados, con varios ejemplares de origen vegetativo.

Las podocarpáceas presentes, Podocarpus nubigenus y Saxegothaea conspicua, con una clara dominancia en densidad de esta última, se presentan al igual que los casos anteriores con bajas densidades (cuadro 2). Donoso (1994) indica que las densidades de plántulas de Saxegothaea conspicua pueden variar, dependiendo del sitio, 
entre 8.000 hasta 9.000 individuos ha-1 ${ }^{-1}$ mientras que en este estudio se registraron solo 3.565 plántulas ha $^{-1}$. Por otra parte, Donoso (1989b) informa que para la Cordillera de la Costa, intervenciones del tipo tala rasa al tercer año gatillan una regeneración de 278 plantas ha $^{-1}$ de Saxegothaea conspicua y 4.000 plantas ha $^{-1}$ de Podocarpus nubigenus, densidades para esta última especie muy distantes de las registradas en el presente estudio. El mayor número de plántulas de Saxegothaea conspicua se registra bajo la cobertura de otras especies, situación que le es propicia y necesaria para lograr un establecimiento seguro, debido a su condición de especie tolerante (Donoso 1989a, Donoso et al. 1999). Estas observaciones permiten inferir la importancia del efecto nodriza en la regeneración de especies arbóreas del bosque siempreverde y de la necesidad de estudiar con mayor detalle estos mecanismos de facilitación.

Importante es destacar que aun cuando el área de estudio colinda con un bosque adulto de Nothofagus nitida, esta especie no presenta una importante número de plántulas y brinzales. Una hipótesis que explicaría esta situación sería la dirección del viento en la época de diseminación (fines de verano e inicio de otoño) del lugar, que trasladaría las semillas aladas de esta especie hacia sectores de laderas noreste o también a la fuerte competencia ejercida por la cobertura de las principales especies arbustivas (ej. Chusquea spp.) y helechos terrestres de grandes frondas (Lophosoria quadripinnata).

\section{CONCLUSIONES}

Los resultados obtenidos evidencian que el bosque siempreverde, afectado por tala rasa, quema y abandono hace 14 años en la Reserva Costera Valdiviana, se encuentra recuperándose naturalmente con las especies nativas propias de la comunidad original. La forma de vida predominante es la fanerófita y la forma de crecimiento principal es la arbórea, siendo Drimys winteri la especie más importante en ambos casos. La presencia de hemicriptófitas, confirma el efecto del disturbio antrópico en el lugar, principalmente en los sectores más abiertos y expuestos a la radiación directa.

Las principales especies arbóreas pioneras son Drimys winteri y Embothrium coccineum, las que se establecieron rápidamente luego del disturbio antrópico. Tanto Amomyrtus luma como Saxegothaea conspicua se establecen vegetativamente en aquellos sectores con luz directa. En condiciones de sombra y a nivel de plántulas, estas especies crecen bajo la cobertura de Chusquea macrostachya y Lophosoria quadripinnata, siendo esta última especie además la que presenta la mayor cobertura relativa del área. La baja cantidad de plántulas y brinzales de Nothofagus nitida en el área de estudio, sugiere una limitación en el proceso de dispersión de sus semillas desde los bosques adyacentes, sumado a las dificultades de establecimiento derivado de la competencia con especies arbustivas previamente establecidas.
Las actuales condiciones de sitio y la relativamente cercana fecha de tala y quema, indican que esta comunidad se encuentra en una etapa sucesional temprana de desarrollo. De excluirse el ganado vacuno, la tasa de recuperación del bosque siempreverde aumentaría y con ello disminuiría el crecimiento y desarrollo de especies introducidas. La evaluación de nuevos sitios, especialmente aquellos no afectados por ganadería y quema de desechos, contribuirá a comprender más acabadamente los patrones sucesionales iniciales de los bosques siempreverdes y recomendar alternativas técnicas apropiadas para la restauración ecológica de estos bosques.

\section{AGRADECIMIENTOS}

Agradecemos a los guardaparques de la Reserva Costera Valdiviana Erwin Obando y Danilo González por sus reportes históricos del área de estudio. A The Nature Conservancy por facilitarnos las dependencias de la Reserva Costera Valdiviana. A Sinuhé Ortega, Marcos Cortés, Lorena Saavedra y Zenón Mardones por su apoyo en terreno. Al Centro de Ciencia del Clima y la Resiliencia (CR) ${ }^{2}$ (CONICYT/FONDAP/15110009) por el financiamiento de esta investigación.

\section{REFERENCIAS}

Alberdi M, C Donoso. 2004. Variación en Embothrium coccineum J.R. et G. Forster (Notro). In Donoso C, L Gallo, A Premoli, R Ipinza eds. Variación intraespecífica en especies arbóreas de los bosques templados de Chile y Argentina. Santiago, Chile. Editorial Universitaria. 420 p.

Braun-Blanquet, J. 1979. Fitosociología, bases para el estudio de comunidades vegetales. Madrid, España. Blume Ediciones. $820 \mathrm{p}$.

Callaway R. 1995. Positive interactions among plants. Botanical Review 61: 306-349.

Centro de Estudios Ambientales. CEA. 2005. Caracterización y zonificación de la cordillera pelada. Proyecto (PIMS 1859) Bosque Templado Valdiviano. 362 p.

CIREN. Publicación CIREN No 123. 2003. Estudio Agrológico X Región. Descripciones de suelos. Materiales y símbolos. Santiago, Chile, CIREN. 412 p. 2 vol.

Di Castri F, E Hajek. 1976. Bioclimatología de Chile. Santiago, Chile. Editorial Universidad Católica de Chile. 128 p.

Donoso C. 1989a. Antecedentes básicos para la silvicultura del tipo forestal siempreverde. Bosque 10(1): 37-53.

Donoso C. 1989b. Regeneración y crecimiento en el tipo forestal siempreverde costero y andino tras distintos tratamientos silviculturales. Bosque 10(2): 69-83.

Donoso C. 1994. Bosques templados de Chile y Argentina. Santiago, Chile. Editorial Universitaria. 484 p.

Donoso C, P Donoso, M González, V Sandoval. 1999. Los bosques siempreverdes. In Donoso C, A Lara eds. Silvicultura de los bosques nativos de Chile. Santiago, Chile. Editorial Universitaria. $421 \mathrm{p}$.

Donoso C, B Escobar, P Donoso, F Utreras. 2006. Drimys winteri J.R. et G. Forster. In Donoso C ed. Las especies arbóreas de los bosques templados de Chile y Argentina. Autoecología. 
Valdivia, Chile. Marisa Cúneo Ediciones. 678 p.

Ellemberg H, D Mueller-Dombois. 1966. A key to Raunkiaer plant life forms with revised subdivisions. Veröffentlichungen des Geobotanischen Institutes der ETH, Stiftung Rübel, Zürich. 37: 56- 73.

Farías A, D Tecklin. 2003. Caracterización preliminar de los predios Chaihuín y Venecia, Cordillera de la Costa, Décima Región. Presentación. Documento No6, serie de publicaciones WWF Chile. Programa Ecoregión Valdiviana. 25 p.

González A. 2000. Evaluación del recurso vegetacional en la cuenca del río Budi, situación actual y propuestas de manejo. Tesis de Licenciatura, Temuco, Chile. Facultad de Ciencias, Universidad Católica de Temuco. 110 p.

Grigera D, C Brion, JO Chiapella, MS Pillado. 1996. Las formas de vida de las plantas como indicadores de factores ambientales. Medio Ambiente 13(1) 11-29.

Hauenstein E, C Ramírez, M Latsague, D Contreras. 1988. Origen fitogeográfico y espectro biológico como medida del grado de intervención antrópica en comunidades vegetales. Medio Ambiente 9: 140-142.

Honorato R. 2000. Manual de Edafología. Cuarta Edición. Santiago, Chile. Ediciones Universidad Católica de Chile. 244 p.

Lara A, C Donoso, J Aravena. 1997. La conservación del bosque nativo de Chile: problemas y desafíos. In Armesto J, C Villagrán, M Arroyo eds. Ecología de los bosques nativos de Chile. Santiago, Chile. Editorial Universitaria. 477 p.

Marticorena C, M Quezada. 1985. Catálogo de la flora vascular de Chile. Gayana Botanica 42: 1- 155.

Muñoz J. 2009. El espinillo (Ulex europaeus L. 1753): un invasor biológico en el sur de chile: estado de su conocimiento y alternativas de control. Gestión Ambiental 17: 23-44.

Myers N, RA Mittermeir, CG Mittermeir, GA Da Fonceca, J Kent. 2000. Biodiversity hotspots for conservation priorities. Nature 403:853-858.

Ramírez C, C San Martín. 2005. Asociaciones vegetales de la Cordillera de la Costa de la Región de Los Lagos. In SmithRamírez C, J Armesto, C Valdovinos eds. Historia, biodiversidad y ecología de los bosques costeros de Chile. San- tiago, Chile. Editorial Universitaria. 708 p.

Sadzawka A, M Carrasco, R Grez, M Mora, H Flores, A Neaman. 2006. Métodos de análisis recomendados para los suelos de Chile. Santiago, Chile. Serie Actas INIA N 34. 164 p.

Schlatter J, R Grez, V Gerding. 2003. Manual para el reconocimiento de suelos. Valdivia, Chile. Universidad Austral de Chile. 143 p.

Soto D, J Bannister, A Ríos, C Le Quesne. 2010. Nuevos registros de poblaciones amenazadas de Pilgerodendron uviferum D. Don (Florin) en su límite norte de la Cordillera de la Costa chilena. Gayana Botanica 67(1): 120-124.

Stokes M, T Smiley. 1996. An introduction to tree-ring dating. The University Arizona Press, Tucson, Arizona, USA. 73 p.

Szejner P, C Le Quesne, ME González, D Christie. 2011. Recuperación de bosques de Pilgerodendron uviferum (D. Don) Florin luego de perturbaciones antrópicas en la Cordillera de la Costa de Valdivia, Chile. Gayana Botanica 68(2): 196-206.

Veblen T, T Kitzberger, R Villalba. 2004. Nuevos paradigmas en ecología y su influencia sobre el conocimiento de la dinámica de bosques del sur de Argentina y Chile. In Arturi M, J Frangi, J Goya eds. Ecología y manejo de bosques nativos de Argentina. La Plata, Argentina. Editorial Universidad Nacional de La Plata. p. 1-48.

Vidal O, A Reif. 2011. Effect of a tourist-ignited wildfire on Nothofagus pumilio forests at Torres del Paine biosphere reserve, Chile (Southern Patagonia). Bosque 32(1): 64-76.

Weinberger P. 1997. Definición de grupos ecológicos en formaciones boscosas siempreverdes de la zona austral de Chile. Bosque 18(2): 29-41.

Wikum D, G Schanholtzer. 1978. Aplication of the Braun-Blanquet cover-abundance scale of vegetation analysis in land development studies. Environmental management 2: 323329.

Zuloaga F, O Morrone, M Belgrano. 2008. Catálogo de las plantas vasculares del Cono Sur (Argentina, sur de Brasil, Chile, Paraguay y Uruguay). Saint Louis, USA. Missuri Botanical Garden Press. 3.348 p. 
Anexo 1. Propiedades físicas del suelo en el área estudiada.

Soil physical properties in the study area.

\begin{tabular}{|c|c|c|c|c|c|c|c|c|c|c|c|c|}
\hline $\mathrm{H}$ & $\mathrm{P}(\mathrm{cm})$ & $\mathrm{F}-\mathrm{N}$ & $\begin{array}{c}\mathrm{CRF} / 100 \\
\mathrm{~cm}^{2}\end{array}$ & E & Cs & $\mathrm{T}$ & Q (\%) & Color & Di & DA $\left(\mathrm{g} \mathrm{cm}^{-3}\right)$ & $\mathrm{RP}\left(\mathrm{kg} \mathrm{cm}^{-2}\right)$ & $\begin{array}{c}\sum \mathrm{CAA} \\
(\mathrm{mm})\end{array}$ \\
\hline \multicolumn{13}{|c|}{ Perfil 1} \\
\hline A & $0-4$ & $\mathrm{O}-\mathrm{C}$ & $\mathrm{Fr}>50$ & G & MF & $\mathrm{FL}$ & $<5$ & 10 YR 3/3 & Mo & $0,85+/-0,06$ & $1,80+/-0,42$ & \\
\hline BA & $4-14$ & O-Gr & D (20-50) & G-Sp & F & FL & $<5$ & $10 \mathrm{YR}$ 4/6 & Mo & $0,88+/-0,11$ & $1,95+/-0,67$ & \\
\hline B & $14-27$ & $\mathrm{I}-\mathrm{C}$ & F (10-20) & Sp & F & FA & 10 & $10 \mathrm{YR} 4 / 6$ & Mo & $0,95+/-0,06$ & $1,88+/-0,34$ & 59 \\
\hline BC & $27-33$ & $\mathrm{I}-\mathrm{Ab}$ & M (5-10) & Sp & $\mathrm{F}$ & - & 50 & $10 \mathrm{YR} 4 / 6$ & Mo & - & - & \\
\hline $\mathrm{C}$ & $33-40+$ & - & 0 & M & Fm & - & $>50$ & - & Lt & - & - & \\
\hline \multicolumn{13}{|c|}{ Perfil 2} \\
\hline A & $0-3$ & $\mathrm{O}$ & $\mathrm{Fr}>90$ & G-Sp & F & FLA & - & 10 YR 2/2 & Mo & $0,98+/-0,07$ & $0,63+/-0,18$ & \\
\hline BA & $3-22$ & $\mathrm{O}$ & $\mathrm{D}(30)$ & $\mathrm{Sp}$ & F-Fm & FLA & $2-3$ & $10 \mathrm{YR} 3 / 6$ & Mo & $1,41+/-0,10$ & $2,33+/-0,44$ & \\
\hline B & $22-34$ & $\mathrm{O}$ & M (9) & M & $\mathrm{F}$ & FA & 50 & $10 \mathrm{YR} 4 / 6$ & Mo & $1,54+/-0,03$ & $3,20+/-0,67$ & 58 \\
\hline BC & $34-64$ & I & $\mathrm{L}(1-2)$ & M & $\mathrm{F}$ & FA & 20 & 10 YR 5/6 & Lt & $1,45+/-0,05$ & $4,03+/-0,51$ & \\
\hline $\mathrm{C}$ & $64-100+$ & - & - & M & $\mathrm{F}$ & $\mathrm{AF}$ & 10 & 10 YR 5/4 & $\mathrm{Lt}$ & - & $4,10+/-0,41$ & \\
\hline
\end{tabular}

$\mathrm{H}=$ horizonte, $\mathrm{P}=$ profundidad, $\mathrm{F}-\mathrm{N}=$ forma y nitidez $(\mathrm{O}=$ ondulado, $\mathrm{C}=$ claro, $\mathrm{Gr}=$ gradual, $\mathrm{I}=$ irregular, $\mathrm{Ab}=$ abrupto $), \mathrm{CRF}=$ contenido de raíces finas $(\mathrm{Fr}$ = fieltro raíces, $\mathrm{D}=$ denso, $\mathrm{F}=$ fuerte, $\mathrm{M}=$ mediano, $\mathrm{L}=$ leve $), \mathrm{E}=$ estructura $(\mathrm{G}=$ granular, $\mathrm{Sp}=$ subpoliédrico, $\mathrm{M}=$ masivo $), \mathrm{Cs}=$ consistencia $(\mathrm{MF}=$ muy friable, $\mathrm{F}=$ friable, $\mathrm{Fm}=$ firme $), \mathrm{T}=$ textura $(\mathrm{FL}=$ franco limosa, $\mathrm{FA}=$ franco arenosa, $\mathrm{FLA}=$ franco, limo y arena, $\mathrm{AF}=$ arena franco $), \mathrm{Q}=$ pedregosidad, $\mathrm{Di}=$ drenaje interno $(\mathrm{Mo}=$ moderado, $\mathrm{Lt}=$ lento $), \mathrm{DA}=$ densidad aparente, $\mathrm{RP}=$ resistencia a la penetración, $\Sigma \mathrm{CCA}=$ capacidad de agua aprovechable total .

Anexo 2. Propiedades químicas del suelo en el área estudiada.

Soil chemical properties in the study area.

\begin{tabular}{|c|c|c|c|c|c|c|c|c|c|c|}
\hline \multirow[b]{2}{*}{ Horizonte } & \multicolumn{5}{|c|}{ Perfil 1} & \multicolumn{5}{|c|}{ Perfil 2} \\
\hline & A & BA & $\mathrm{B}$ & $\mathrm{BC}$ & $\mathrm{C}$ & A & BA & $\mathrm{B}$ & $\mathrm{BC}$ & $\mathrm{C}$ \\
\hline $\mathrm{pH}$ (agua) & $4,86 \mathrm{a}$ & $4,71 \mathrm{a}$ & $4,99 \mathrm{a}$ & $4,86 \mathrm{a}$ & - & $4,92 \mathrm{a}$ & $4,22 \mathrm{a}$ & $4,72 \mathrm{a}$ & $4,72 \mathrm{a}$ & $4,87 \mathrm{a}$ \\
\hline $\mathrm{pH}(\mathrm{KCl})$ & $3,70 \mathrm{a}$ & $3,72 \mathrm{a}$ & $3,87 \mathrm{a}$ & $3,90 \mathrm{a}$ & - & $3,72 \mathrm{a}$ & $3,42 \mathrm{a}$ & 3,59 a & $3,77 \mathrm{a}$ & 3,97 a \\
\hline $\mathrm{Ct}(\%)$ & $6,89 \mathrm{~d}$ & $4,99 \mathrm{c}$ & $4,11 \mathrm{c}$ & - & - & $10,81 \mathrm{e}$ & $5,79 \mathrm{~d}$ & $1,61 \mathrm{c}$ & - & - \\
\hline Nt (\%) & $0,17 \mathrm{c}$ & $0,14 \mathrm{~b}$ & $0,11 \mathrm{~b}$ & - & - & $0,31 \mathrm{c}$ & $0,19 \mathrm{c}$ & $0,06 \mathrm{a}$ & - & - \\
\hline $\mathrm{C} / \mathrm{N}$ & 40,13 e & $35,71 \mathrm{~d}$ & $35,88 \mathrm{~d}$ & - & - & $34,96 \mathrm{~d}$ & $30,95 \mathrm{~d}$ & 26,62 c & - & - \\
\hline $\mathrm{P}$ (Olsen) (mg kg-1) & $2,60 \mathrm{a}$ & $1,40 \mathrm{a}$ & $1,40 \mathrm{a}$ & - & - & $2,80 \mathrm{a}$ & $1,40 \mathrm{a}$ & $1,60 \mathrm{a}$ & - & - \\
\hline $\mathrm{K}\left(\mathrm{mg} \mathrm{kg}^{-1}\right)$ & $151 \mathrm{c}$ & $98 \mathrm{c}$ & $55 \mathrm{~b}$ & - & - & $197 \mathrm{~d}$ & $56 \mathrm{~b}$ & $14 \mathrm{a}$ & - & - \\
\hline $\mathrm{K}\left(\mathrm{cmol}+\mathrm{kg}^{-1}\right)$ & 0,39 c & $0,25 \mathrm{c}$ & $0,14 \mathrm{~b}$ & - & - & $0,50 \mathrm{~d}$ & $0,14 \mathrm{a}$ & $0,04 \mathrm{a}$ & - & - \\
\hline Ca $\left(\mathrm{mg} \mathrm{kg}^{-1}\right)$ & $250 \mathrm{c}$ & 32 a & $15 \mathrm{a}$ & - & - & 1160 e & $53 \mathrm{~b}$ & $50 \mathrm{~b}$ & - & - \\
\hline $\mathrm{Ca}\left(\mathrm{cmol}+\mathrm{kg}^{-1}\right)$ & $1,25 \mathrm{c}$ & 0,16 a & 0,07 a & - & - & 5,80 e & $0,27 \mathrm{~b}$ & $0,25 \mathrm{~b}$ & - & - \\
\hline $\operatorname{Mg}\left(\mathrm{mg} \mathrm{kg}^{-1}\right)$ & $67 c$ & $24 \mathrm{a}$ & $16 \mathrm{a}$ & - & - & $236 \mathrm{~d}$ & $22 \mathrm{a}$ & $16 \mathrm{~b}$ & - & - \\
\hline $\mathrm{Mg}\left(\mathrm{cmol}+\mathrm{kg}^{-1}\right)$ & $0,55 \mathrm{c}$ & $0,20 \mathrm{a}$ & 0,13 a & - & - & $1,94 \mathrm{~d}$ & 0,18 a & 0,13 a & - & - \\
\hline $\mathrm{Na}\left(\mathrm{mg} \mathrm{kg}^{-1}\right)$ & $64 \mathrm{~b}$ & 37 a & $25 \mathrm{a}$ & - & - & $64 \mathrm{~b}$ & $25 \mathrm{a}$ & $22 \mathrm{a}$ & - & - \\
\hline $\mathrm{Na}\left(\mathrm{cmol}+\mathrm{kg}^{-1}\right)$ & $0,28 \mathrm{~b}$ & $0,16 \mathrm{~b}$ & $0,11 \mathrm{a}$ & - & - & $0,28 \mathrm{~b}$ & $0,11 \mathrm{a}$ & 0,09 a & - & - \\
\hline $\mathrm{Al}$ (intercambiable) $\left(\mathrm{mg} \mathrm{kg}^{-1}\right)$ & $190 \mathrm{e}$ & $216 \mathrm{e}$ & $157 \mathrm{e}$ & - & - & 113 e & $427 \mathrm{e}$ & 179 e & - & - \\
\hline $\mathrm{Al}$ (intercambiable) $\left(\mathrm{cmol}^{+} \mathrm{kg}^{-1}\right)$ & $2,11 \mathrm{e}$ & $2,41 \mathrm{e}$ & $1,75 \mathrm{e}$ & - & - & $1,25 \mathrm{e}$ & $4,74 \mathrm{e}$ & 1,99 a & - & - \\
\hline Suma de bases $\left(\mathrm{cmol}+\mathrm{kg}^{-1}\right)$ & $2,47 \mathrm{a}$ & $0,77 \mathrm{a}$ & 0,45 a & - & - & $8,52 \mathrm{c}$ & $0,70 \mathrm{a}$ & $0,52 \mathrm{a}$ & - & - \\
\hline Saturación de aluminio (\%) & 46,13 e & 75,76 e & 79,42 e & - & - & $12,79 \mathrm{~d}$ & 87,22 e & 79,44 a & - & - \\
\hline $\mathrm{Fe}\left(\mathrm{mg} \mathrm{kg}^{-1}\right)$ & 363,20 e & 365,40 e & $275,30 \mathrm{e}$ & - & - & 421,70 e & 363,10 e & 230,60 e & - & - \\
\hline $\operatorname{Mn}\left(\mathrm{mg} \mathrm{kg}^{-1}\right)$ & $6 \mathrm{~b}$ & 4 a & $1 \mathrm{a}$ & - & - & $45 \mathrm{~d}$ & $2 \mathrm{a}$ & $1 \mathrm{a}$ & - & - \\
\hline $\mathrm{Cu}\left(\mathrm{mg} \mathrm{kg}^{-1}\right)$ & $1,50 \mathrm{~b}$ & $1,50 \mathrm{~b}$ & $1,3 \mathrm{~b}$ & - & - & $1,40 \mathrm{~b}$ & $1,0 \mathrm{~b}$ & $1,00 \mathrm{~b}$ & - & - \\
\hline $\mathrm{Zn}\left(\mathrm{mg} \mathrm{kg}^{-1}\right)$ & $1,60 \mathrm{c}$ & $1,00 \mathrm{c}$ & $0,80 \mathrm{c}$ & - & - & $5,80 \mathrm{e}$ & $1,10 \mathrm{c}$ & $0,90 \mathrm{c}$ & - & - \\
\hline $\mathrm{S}\left(\mathrm{mg} \mathrm{kg}^{-1}\right)$ & $16,30 \mathrm{~d}$ & $12,43 \mathrm{c}$ & $14,68 \mathrm{~d}$ & - & - & $14,48 \mathrm{~d}$ & $11,28 \mathrm{c}$ & $15,50 \mathrm{~d}$ & - & - \\
\hline $\mathrm{B}\left(\mathrm{mg} \mathrm{kg}^{-1}\right)$ & $1,36 \mathrm{~d}$ & $1,60 \mathrm{~d}$ & $1,42 \mathrm{~d}$ & - & - & $1,36 \mathrm{~d}$ & $1,74 \mathrm{~d}$ & $0,84 \mathrm{c}$ & - & - \\
\hline $\mathrm{Al}$ (extraíble) $\left(\mathrm{mg} \mathrm{kg}^{-1}\right)$ & $676 \mathrm{c}$ & $800 \mathrm{c}$ & 802 c & $609 \mathrm{c}$ & - & 598 c & 889 c & $457 \mathrm{~b}$ & $207 \mathrm{a}$ & $344 \mathrm{~b}$ \\
\hline
\end{tabular}

$\mathrm{a}=$ muy bajo, $\mathrm{b}=$ bajo, $\mathrm{c}=$ medio, $\mathrm{d}=$ alto, $\mathrm{e}=$ muy alto. 
